\title{
QUAND UN ÉCRIVAIN FRANÇAIS PERD LE NORD : DRIEU LA ROCHELLE ET L'ESTHÉTISATION FASCISTE
}

\author{
GuilLAUme BRIDET*
}

L'hypothèse selon laquelle il y aurait un abîme infranchissable entre la littérature et la culture, d'un côté, et le fascisme, de l'autre ${ }^{1}$, outre qu'elle offre un argument précieux aux thuriféraires contemporains des écrivains fascistes soucieux de redonner une nouvelle vie à leur œuvre en la séparant des déclarations politiques dont ils se sont rendus coupables, présente également le défaut majeur de rendre incompréhensible la manière dont certains écrivains, dont Drieu La Rochelle à partir de 1934, ont pu adhérer à une telle idéologie. Discours politiques et œuvres de fiction ne sont pas complètement étrangers l'un à l'autre. Il est ainsi possible de traquer la manière dont le discours fasciste est relayé, dans l'œuvre romanesque de Drieu, par celui des divers narrateurs ou personnages identifiés comme ses porte-parole, et de dégager la présence indéniable d'une thématique raciste. Le Juif, la femme et l'homosexuel, auxquels on peut le plus souvent ajouter l'intellectuel, sont autant de figures inquiétantes, voire détestées, incarnant la décadence contemporaine ; l'homme nordique, descendant des vagues d'invasion aryennes, héritier de l'âge d'or médiéval et implanté essentiellement dans les pays de langue germanique, leur oppose son courage guerrier et sa noblesse d'âme.

\footnotetext{
* Paris 13/CENEL.

1. Cette lecture est prégnante dans la réception américaine de l'œuvre de Drieu La Rochelle (voir Richard J. Golsan, « Drieu La Rochelle aux États-Unis : entre l'esthétique et le fascisme », dans Marc Dambre (dir.), Drieu La Rochelle, écrivain et intellectuel, Actes du colloque international organisé par le centre de recherche «Études sur Nimier », Sorbonne nouvelle, 9 et 10 décembre 1993, Paris, Presses de la Sorbonne nouvelle, 1995, p. 65-75).
}

RHLF, 2009, $\mathrm{n}^{\circ} 3$, p. 661-680 
Envisager le lien du fascisme et de la littérature ne doit cependant pas conduire à faire de l'œuvre littéraire le simple reflet de prises de position politiques. S'il y a bien, comme le soulignent Jean-Luc Nancy et Philippe Lacoue-Labarthe, "une logique du fascisme ", ce qui revient à dire qu' « une certaine logique est fasciste $»^{2}$, il faut aussi considérer que cette logique n'apparaît pas de la même manière dans les discours qui prennent directement pour objet la réalité et dans les récits fictifs. Ce n'est pas que ceux-ci soient moins logiques que ceux-là, mais les uns et les autres n'obéissent pas aux mêmes contraintes sociales et n'apportent pas de la même manière une réponse aux désirs individuels de leurs auteurs. Le refus de réduire l'œuvre littéraire à l'expression transparente d'une opinion politique, qui vaut pour l'ensemble des individus ayant mené une double carrière d'écrivain et de propagandiste, vaut a fortiori pour les auteurs fascistes. Si, comme l'affirme Benjamin, le fascisme consiste essentiellement en « une esthétisation de la vie politique $»^{3}$, il y a en effet un intérêt particulier à scruter les diverses modalités selon lesquelles la démarche littéraire de Drieu n'est ni purement esthétique, ni purement politique, mais constitue le lieu privilégié d'une recherche esthétique éminemment politique.

Il apparaît tout d'abord que c'est par le biais de la fiction littéraire et non par celui de l'essai politique que Drieu s'efforce dans un premier temps de préciser une représentation du monde fondée sur la guerre des races. Alors que, dans les essais qu'il publie avant 1934, il prend position contre le nationalisme et en faveur d'un fédéralisme européen, ses œuvres romanesques publiées dans le même temps constituent un imaginaire alternatif grâce auquel il s'approprie peu à peu la thématique raciste. L'esthétisation du politique s'entend d'abord ici comme élaboration d'une pensée politique dans un cadre littéraire qui certes n'est pas absolument autonome, puisque Drieu se nourrit aussi de la lecture des maîtres de l'idéologie raciste, mais qui annonce et anticipe par de multiples traits la conversion ultérieure à cette idéologie.

La logique que Drieu met ensuite en œuvre dans ses récits fictifs qu'on peut qualifier de fascistes n'est pas la même que celle d'un idéologue comme Hitler. Chez ce dernier, la logique est celle d'un délire qui interprète le monde entier selon une grille de lecture sans faille : d'un côté, le Juif est l'incarnation du mal et doit être exterminé ; de l'autre, le nordique est le sauveur de l'Allemagne et doit imposer une domination

2. Philippe Lacoue-Labarthe, Jean-Luc Nancy, Le Mythe nazi, Paris, Éditions de l'Aube, coll. Monde en cours - Intervention philosophique, 1991, p. 25.

3. Walter Benjamin, «L'œuvre d'art à l'époque de sa reproductibilité technique » [dernière version, 1939], Euvres, tome III, traduit de l'allemand par Maurice de Gandillac, Rainer Rochlitz et Pierre Rusch, Paris, Gallimard, coll. Folio/Essais, 2000, p. 314. 
universelle. L'imaginaire de Drieu est certes, lui aussi, fortement polarisé entre figure de la corruption et figure de l'idéal, mais la rationalisation délirante de la guerre des races ne s'impose pas au point de masquer totalement un doute identitaire profond. À la logique binaire de la condamnation de la race maudite et de l'exaltation de la race salvatrice s'ajoute donc le plus souvent l'auto-dénigrement de la race française à laquelle il appartient. L'œuvre littéraire, sans doute moins contrôlée que le discours idéologique, témoigne ici d'une impossibilité d'envisager une idéalisation effective de son identité raciale.

Cette idéalisation de soi est plus spécialement impossible dans un cadre littéraire, parce qu'il y a une contradiction entre l'identité nordique et l'identité de l'écrivain, entre celle du héros hitlérien manieur de foules et celle du pauvre manieur de mots. Si la plupart des récits fictifs de Drieu sont largement inspirés de sa propre vie et forment une sorte d'autobiographie romancée chargée d'idéaliser sa vie en la conformant à l'idéal esthétique de l'homme nordique et du poème épique, la création romanesque demeure, à ses yeux, le fait d'un homme dont la force de vie est affaiblie et qui ne peut donc jamais atteindre l'idéal auquel il aspire. L'esthétisation idéalisante laisse ainsi très largement la place à une chronique satirique et désenchantée de la décadence.

Avant 1934, on observe un contraste flagrant entre les positions politiques qu'assume Drieu dans ses essais et celles qui affleurent plus ou moins explicitement dans ses œuvres de fiction. Dans Mesure de la France (1922), Genève ou Moscou (1928) ou encore L'Europe contre les patries (1931), l'essayiste se montre, à quelques points près, d'une remarquable constance. Internationaliste convaincu dénigrant les nationalismes français et allemand, Drieu appelle les vieilles nations européennes, notablement affaiblies depuis la Grande Guerre, à se fédérer sous l'égide de la SDN afin de ne pas subir la domination des empires américain et russe. «L'Europe se fédérera ou elle se dévorera, ou elle sera dévorée » ${ }^{4}$. Drieu admire les peuples anglo-saxons et particulièrement le peuple anglais, qui présente l'avantage d'être européen et que les habitants du continent peuvent par conséquent prendre pour modèle. Les Anglais ont à ses yeux le sens de la grandeur et ils mettent à l'épreuve leur virilité par une pratique sportive assidue. Leur réussite n'est pas seulement fondée, comme celle des États-Unis, sur la puissance économique ; elle est aussi celle d'esprits et de corps que caractérisent la beauté et la distinction ${ }^{5}$. Drieu ne croit en

4. Pierre Drieu La Rochelle, Mesure de la France, Paris, Bernard Grasset, 1922, p. 65.

5. Sur le rapport de Drieu avec l'Angleterre, voir Douglas Gallagher, «Influences anglaises », dans Marc Hanrez (dir.), Pierre Drieu La Rochelle, Paris, Éditions de l'Herne, coll. Les Cahiers de l'Herne, 1982, p. 337-354. 
revanche pas en la Russie soviétique qui, selon lui, cherche à se moderniser selon des principes capitalistes camouflés ${ }^{6}$. Les socialistes ne sont pas l'avant-garde d'une classe ouvrière qui n'existe pas, mais seulement des bourgeois en puissance. C'est la raison pour laquelle Drieu dénonce, comme il l'écrit aux surréalistes en 1925, « ce vieux cliché clinquant de la révolution qui roule d'est en ouest $»^{7}$. Récusant aussi bien Moscou à l'est, que New York à l'ouest, les Européens doivent avant tout penser à s'unir en prenant pour exemple l'Angleterre.

On ne retrouve dans les œuvres de fiction de Drieu, ni les mêmes prises de position politiques, ni la même univocité. Trois distorsions majeures sont à souligner : une relégation de l'idéal européen au profit des États-Unis et de la Russie; une ambivalence évidente à l'encontre du modèle anglo-saxon et, plus précisément, de l'Angleterre ; et une présence inédite, puisqu'ils ne sont pratiquement pas mentionnés dans les essais, des pays méditerranéens.

Loin d'être des Européens convaincus, certains personnages des romans de Drieu envisagent d'abord la Russie soviétique et les États-Unis comme des pays d'avenir. Blèche, le personnage féminin du roman éponyme, décide de partir vivre de l'autre côté de l'Atlantique et elle s'installe à New York. Boutros, héros d'Une femme à sa fenêtre et communiste un peu particulier dans ses admirations atlantiques, affirme quant à lui n'avoir «plus confiance que dans les Américains ou les Russes $»^{8}$. L'auteur de fiction semble ici exorciser les doutes d'un essayiste qui veut se persuader, sans trop y croire, que l'Europe a encore un avenir. Si le narrateur de L'Homme couvert de femmes se félicite de ce que les rapports entre les personnages anglais et français ne soient « guère affectés par le nationalisme outré qui domine à certains étages de la société $»^{9}$, il n'en attribue pas moins à chacun d'entre eux des caractéristiques nationales qui laissent mal augurer de leur entente profonde. La caractérisation physique et morale des personnages en des termes renvoyant à leur origine nationale, ponctuelle dans ce premier roman de 1925, s'amplifie d'ailleurs notablement à partir du troisième roman de Drieu, Une femme à sa fenêtre, publié quatre ans plus tard. Lui-même semble faire sienne la pen-

6. Sur la critique du communisme par Drieu, voir Jean-Pierre Morel, « Drieu La Rochelle et le communisme », dans Marc Dambre (dir.), Drieu La Rochelle, écrivain et intellectuel, op. cit., p. 53-64.

7. Pierre Drieu La Rochelle, « La véritable erreur des surréalistes » [NRF, $\mathrm{n}^{\circ} 143$, août 1925], Sur les écrivains [1964], Essais critiques réunis, préfacés et annotés par Frédéric Grover, Paris, Gallimard, Nouvelle édition, 1982, p. 46.

8. Pierre Drieu La Rochelle, Une femme à sa fenêtre [1929], Paris, Gallimard, coll. Folio, 1996, p. 172.

9. Pierre Drieu La Rochelle, L'Homme couvert de femmes [1925], Paris, Gallimard, coll. L'Imaginaire, 1994, p. 36. 
sée du narrateur d'une de ses nouvelles : « les personnalités n'existent que comme réfraction d'un peuple $»^{10}$. Cette caractérisation, qui ne fait que s'accentuer au fil de l'œuvre, n'est pas sans mettre en cause les possibilités d'un accord entre les peuples européens.

Autre changement notable, lié à ce dernier point, l'admiration des pays anglo-saxons et surtout de l'Angleterre laisse la place à une certaine ambivalence qui met cette fois en péril ce qui pourrait demeurer de l'ancienne Entente cordiale. Dans L'Homme couvert de femmes, si Gille, double romancé de Drieu, manifeste " un labyrinthe d'habiletés aimables audevant des Anglais et des Américains qu'il [mêle] un peu », il n'en regrette pas moins l'influence de la civilisation anglo-saxonne : " la morale est fournie au monde aujourd'hui par les Anglo-Saxons, baignoires, lavabos, les gens n'ont plus que cette activité spirituelle : se laver $»^{11}$. À cette dénonciation d'un hygiénisme qui emprisonne le corps et bride les instincts, Drieu ajoute celle d'un matérialisme tout aussi néfaste. Comme il le déclare à l'Anglais Lord Owen, le Gille de Drôle de voyage n'épouse pas sa fille Béatrix parce qu'il ne veut pas se lier avec «la société fondée sur l'argent », société sans grandeur d'âme dont on ne saurait trop dire, à la lecture, si elle caractérise l'Angleterre anglo-saxonne dans son ensemble ou si elle trouve son origine - et l'incertitude ouvre des horizons imaginaires dans lesquels Drieu va bientôt s'engouffrer — dans le fait que lady Owen soit «une Juive $»^{12}$. Matérialisme, productivisme, uniformisation, tous ces traits du capitalisme anglo-saxon empêchent Drieu de donner un plein acquiescement à une civilisation qui n'est pas simplement le fait de l'Angleterre, mais de l'ensemble des pays industrialisés. De la même manière que les bourgeois ne pensent qu'à amasser de l'argent, les « foules du Nord », qu'il désigne assez vaguement par cette seule orientation septentrionale, ne sont bonnes qu'à «se ruer vers les usines $»^{13}$. Le sportif anglo-saxon vanté dans les essais laisse la place à un homme dégradé comptant ses pièces d'or ou qu'on paye à la pièce.

Fait important, enfin, les pays situés autour de la Méditerranée, pourtant largement absents de la réflexion politique de Drieu dans les essais, tiennent une large place dans ses œuvres de fiction. D'un côté, le Sud est valorisé et s'oppose explicitement au Nord industriel comme un espace originel où les hommes n'ont pas encore été pervertis par le machinisme et dans lequel ils demeurent au contact de leurs instincts. Boutros admire

10. Pierre Drieu La Rochelle, "Le déserteur » [1933], La Comédie de Charleroi [1934], Paris, Gallimard, Préface de Julien Hervier, coll. Folio, 1982, p. 218.

11. Pierre Drieu La Rochelle, L'Homme couvert de femmes, op. cit., respectivement p. 35 et 172.

12. Pierre Drieu La Rochelle, Drôle de voyage [1933], Paris, Gallimard, 1978, respectivement p. 317 et p. 58 .

13. Ibid., p. 119. 
les paysans grecs qu'il croise en voiture et en lesquels il voit « une petite troupe d'evzones » ou encore de « grands bergers-soldats $»^{14}$. Leurs qualités sont autant morales que physiques. Ces pâtres font preuve d' " une noble bonté » et sont en outre «superbes : grands et velus $»^{15}$. L'homme des champs dans un pays encore rural s'oppose à l'homme des villes comme un homme proche de la nature à un autre que « la sénile abstraction du Nord $»^{16}$ a coupé de ses racines vitales. On comprend pourquoi, avec l'empire colonial, l'espace méditerranéen offre aux hommes du Nord la possibilité d'une régénération hérö̈que. Dans Rêveuse bourgeoisie, Yves Le Pesnel s'engage dans les Chasseurs d'Afrique afin d'échapper à son destin de petit bourgeois et il retrouve en Algérie et au Maroc la virilité guerrière et sexuelle qui lui faisait défaut à Paris.

Les pays méditerranéens sont cependant victimes d'une décadence séculaire et ont abandonné leur puissance aux nations industrielles. Comme l'Italie dans « La voix » et l'Espagne dans Drôle de voyage soumises au regard cruel de Gille, la Grèce, aux yeux de Boutros, est « inerte, vidée à jamais » et a perdu «toute sa force $»^{17}$. «Tel est l'Esprit. C'est une bête d'acier, une chimère qui traverse les contrées de la terre $\gg^{18}$. L'esprit qui se déplace ici n'est pas inspiré de Hegel, mais, quoique Drieu n'en ait pris connaissance que d'une manière diffuse ${ }^{19}$, des théories de Spengler sur la croissance et le déclin organiques des civilisations. Les vieilles civilisations méditerranéennes ont connu un passé brillant, mais elles croulent à présent sous le poids d'une histoire morte qui empêche toute créativité nouvelle. Lorsque la guerre de 1914 éclate, Yves Le Pesnel revient d'Afrique avec son régiment et, rapidement blessé, il meurt de la gangrène, de cette gangrène morale et industrielle venue du Nord et remplaçant le corps à corps hérö̈que de deux adversaires par l'écrasement de l'ennemi sous les bombes. Le modèle capitaliste et industriel anglosaxon s'impose dans les tranchées et bientôt sur l'ensemble de la planète pacifiée. Les foules du Sud telles qu'elles apparaissent à Gille alors qu'il assiste à une corrida à Grenade sont finalement comparables à celles du Nord.

Cette foule échappée de l'usine la veille y retournerait le lendemain [...]. C'était une foule moderne comme n'importe quelle autre [...]. Sous le grand soleil

14. Pierre Drieu La Rochelle, Une femme à sa fenêtre, op. cit., p. 182.

15. Ibid., p. 162.

16. Pierre Drieu La Rochelle, Drôle de voyage, op. cit., p. 120.

17. Pierre Drieu La Rochelle, Une femme à sa fenêtre, op. cit., p. 159-160.

18. Ibid., p. 165.

19. Sur les limites de l'influence de Spengler sur Drieu et sur son hostilité à Hegel et à Marx, voir Julien Hervier, Deux individus contre l'histoire : Drieu La Rochelle et Ernst Jünger, Paris, Éditions Klincksieck, coll. Bibliothèque du Xxe siècle, 1978, p. 264-272. 
qui la fouillait comme pour y chercher l'occasion d'étinceler, elle paraissait même plus opaque et plus rébarbative qu'une foule du Nord ${ }^{20}$.

La rêverie méditerranéenne d'un espace livré à la force de l'instinct et à la noblesse de l'âme tourne court.

Alors que ses essais d'avant 1934 se contentent de répéter ses appels à l'union de l'Europe et son admiration pour le modèle anglo-saxon tel que l'incarne l'Angleterre, les fictions de Drieu se montrent plus hésitantes. Ni le Nord-Ouest anglo-saxon, ni l'Est communiste, ni même le Sud méditerranéen ne conviennent absolument au Drieu romancier. Contrairement à son œuvre d'essayiste, son œuvre fictionnelle porte les traces d'une errance idéologique qui est aussi une errance imaginaire dans différents espaces géographiques. Au nord-ouest et à l'est, on trouve la puissance politique, mais un matérialisme dégradant; au sud, on trouve l'instinct individuel, mais une décadence collective insoutenable. Si on ne peut affirmer que c'est la réflexion conduite dans le cadre de la fiction qui va conduire à un changement de perspective, la constitution d'un imaginaire géographique alternatif constitue cependant la littérature comme un laboratoire idéologique chargé d'objectiver certaines exigences pulsionnelles demeurées insatisfaites dans le cadre de la prose discursive de l'essai. L'appropriation progressive d'un imaginaire structuré par l'opposition des peuples et l'obsession de la force individuelle autant que collective prend d'abord place dans le domaine de la fiction avant de cristalliser avec l'adhésion à l'idéologie fasciste, de plus en plus accentuée à partir de 1934.

Drieu commence en effet peu à peu à faire siennes les théories distinguant les différents peuples à partir de fondements biologiques. Développées essentiellement en Allemagne à partir de la lecture de La Germanie de Tacite et de la découverte des langues indo-européennes, ces théories opposent essentiellement les peuples sémites originaires du Sud aux peuples aryens, d'origine asiatique et dont les Germains seraient, par le biais des migrations venues du Nord de l'Europe, les dépositaires privilégiés $^{21}$. Avant de constituer le fondement du national-socialisme, l'idée d'une guerre des races trouve un épanouissement tout particulier dans la seconde moitié du XIX ${ }^{\mathrm{e}}$ siècle chez des auteurs français comme Renan, Gobineau et Drumont que Drieu a lus et dont il se fait à présent l'écho :

Les groupements ethniques qui peuplent l'Europe ne sont pas de la même souche que les groupes ethniques qu'on appelle les sémites. Il n'y a ni similitude

20. Pierre Drieu La Rochelle, Drôle de voyage, op. cit., p. 119-120.

21. Pour un historique complet des théories racistes modernes opposant, à partir de critères pseudo-scientifiques, la race nordique aux autres races plus ou moins dégénérées, voir Léon Poliakov, Le Mythe aryen. Essai sur les sources du racisme et des nationalismes [1971], Paris, Pocket, coll. Agora, 1994. 
de caractères physiques, ni similitude de langues. Ce fait très simple et très gros est suffisant pour donner un fondement sûr et irréductible à un minimum de réflexion raciste et à le rendre valable pour tous les peuples d'Europe à l'égard des Juifs ${ }^{22}$.

Chacune des deux races est caractérisée par son sang et correspond à un type humain; le métissage détruit la qualité de la race ; l'Aryen, homme de la noblesse et du courage, doit donc veiller à ne pas se laisser abâtardir ou même envahir par le Sémite âpre au gain et profiteur.

La race nordique, incarnation la plus pure de la race aryenne, est présente dans tout le Nord de l'Europe, en Angleterre donc, en laquelle Drieu continue de voir par intermittence un modèle, mais particulièrement en Allemagne où elle a moins qu'ailleurs été contaminée par la race sémite. Dans Drôle de voyage, publié en 1933, Gille se sentait « du Nord-Ouest de l'Europe $»^{23}$; dans Les Chiens de paille, publié en 1944, Constant Trubert se définit significativement de manière plus restrictive comme «nordique ${ }^{24}$. Ni Nord-Ouest capitaliste ni Est communiste, ni droite ni gauche, l'échappée septentrionale inscrit clairement la pensée de Drieu dans le cadre d'une pensée fasciste. C'est dans ce contexte qu'il faut comprendre la reprise, essentiellement à partir de La Comédie de Charleroi, en 1934, des poncifs de la littérature antisémite et, plus largement, la présence de généralisations qui transforment les personnages en prototypes des races nordique ou juive. Alors que la figure du Juif concentre en elle tout le matérialisme et l'intellectualisme dénoncés par Drieu et devient le ferment de la décadence occidentale, la figure du nordique reconduit l'homme vers une pureté native qui est aussi une promesse de renouveau mêlant indistinctement force de l'instinct et élévation spirituelle.

En passant d'une admiration relative de la puissance anglo-saxonne à une admiration totale de la pureté raciale nordique, Drieu bascule d'un jugement fondé sur des considérations historiques, politiques et économiques à un jugement fondé essentiellement sur des considérations mythiques. «Le sang, si, j'y crois, parce que c'est un mythe. J'aime les mythes $»^{25}$, proclame Constant Trubert. Que l'Allemagne s'affirme au fil des années 1930 comme la puissance dominante en Europe continentale et en vienne à menacer l'empire maritime anglais a certes son importance. Mais c'est surtout le cadre de pensée de Drieu qui change. La puissance de l'Allemagne, dorénavant, est moins, à ses yeux, le fait de son développement industriel et militaire ou de la croissance de sa population que

22. Pierre Drieu La Rochelle, « À propos du racisme » [L'Émancipation nationale, $\mathrm{n}^{\circ} 109$, 29 juillet 1938], Chronique politique. 1934-1942, Paris, Gallimard, 1943, p. 155.

23. Pierre Drieu La Rochelle, Drôle de voyage, op. cit., p. 136.

24. Pierre Drieu La Rochelle, Les Chiens de paille [1964], Paris, Gallimard, coll. L'Imaginaire, 1998, p. 47.

25. Ibid., p. 97. 
d'une pureté raciale constituant, en dernière instance, l'explication de sa supériorité sur les autres nations judaïsées ${ }^{26}$. Ce n'est plus sa civilisation qui définit un peuple, mais la race de ce peuple qui détermine sa civilisation. Ne privilégiant plus qu'un pôle nordique défini comme essentiellement germanique, Drieu se donne une géographie imaginaire correspondant à son désir, puisqu'elle rassemble en elle, à travers le destin de la race allemande, la puissance politique et l'instinct individuel séparés dans ses premières fictions.

Si cette homogénéisation des pôles permet de séparer, d'un côté, toute l'admiration et, de l'autre, toute la haine, et met donc un terme à l'ambivalence dont témoignait le contraste entre les premiers essais et les premières fictions de Drieu, elle fait cependant naître un doute que ne laisse pas transparaître le raidissement idéologique d'un essayiste consacrant la plupart de ses écrits à une exaltation de la pureté et de la virilité nordiques, mais qui est en revanche très présent dans ses œuvres romanesques. Tout le problème qui, sous le rapport de l'identité raciale, se pose à Drieu est d'être né en France, pays situé à mi-chemin du Nord et du Sud et, par conséquent, lieu privilégié de la guerre des races - et de leur mélange néfaste.

Au premier abord, Marseille apparaît au soldat prêt à s'embarquer pour les Dardanelles comme « une ville de boulevards gris, une ville du Nord où il fait chaud en été », mais « en y regardant de plus près », il est bien contraint de remarquer la présence « de drôles de gueules»: «C'était plein de nègres, de Chinois, d'Hindous, de Levantins, de gens nés dans le tunnel entre les deux tropiques de parents inconnus $»^{27}$. Le danger est ici que le peuple français se dissolve dans la France cosmopolite de la même manière que le « régiment français » est « complètement dissous dans Marseille $»^{28}$. La cité phocéenne, fondée par les Grecs et plus tard occupée par les Romains, est l'emblème de cette France du Sud menaçante pour la France du Nord. Dans une nouvelle tardive, le narrateur exprime une certaine rancune à l'égard « des Romains » :

26. Le fondement racial de la puissance est si important pour Drieu qu'il en vient dans les dernières années de la guerre, avec les défaites répétées de l'armée allemande, à insister sur le caractère nordique des vainqueurs anglais du jour (voir Pierre Drieu La Rochelle, « L'Europe socialiste » [La Révolution nationale, 15 avril 1944], dans Marc Hanrez (dir.), Pierre Drieu La Rochelle, op. cit., p. 122). À un Drieu germanophile succède même un ultime slavophile pour lequel la victoire prévisible de l'Armée rouge sur le front de l'Est est la preuve, non pas du caractère profondément mortifère de la volonté de puissance nazie ou du caractère aberrant des mythes racistes, mais d'une supériorité raciale du peuple slave sur le peuple germanique (voir Pierre Drieu La Rochelle, Les Chiens de paille, op. cit., p. 46, p. 93 et p. 119).

27. Pierre Drieu La Rochelle, «Le voyage des Dardanelles » [1933], La Comédie de Charleroi, op. cit., respectivement p. 140 et p. 149.

28. Ibid., p. 149. 
Je m'étonne et je me choque qu'ils aient dérangé le destin nordique de la Gaule, de la Germanie ; je m'en console en pensant qu'ils étaient malgré tout de souche aryenne et que le seul vrai mal qu'ils nous aient apporté c'est celui qu'ils avaient eux-mêmes reçu d'un Orient qui s'était corrompu ${ }^{29}$.

Cette tentative maladroite d'identifier l'héritage latin à l'héritage germanique dans une même origine aryenne et de rejeter le ferment de décadence sur l'Orient est tout à fait caractéristique d'un courant de pensée pour lequel « l'incertaine généalogie des Français » entre une terre galloromaine et des maîtres germaniques forme « une sorte de traumatisme originel ${ }^{30}$ d'autant plus difficile à apaiser qu'elle sert aussi, de l'autre côté du Rhin, à légitimer l'impérialisme d'une prétendue race allemande.

Ce trouble identitaire est si fort qu'il atteint même les personnages romanesques créés par Drieu et censés incarner par excellence l'identité nordique de la France. Gagnant la province normande aux forêts plusieurs fois centenaires afin de retrouver la source pure de la race et la perfection des origines, Gilles constate douloureusement que les paysans de son enfance n'ont plus d'autres recours qu' « un lucre et un alcool maniaques », ne savent plus entendre « la leçon de la nature » et ne sont plus que de « chétifs bourgeois ${ }^{31}$. Cette décadence normande bouleverse à tel point Drieu que, choisissant de décrire la décadence d'une famille bourgeoise dans Rêveuse bourgeoisie, il choisit la famille normande des Le Pesnel.

Menacée jusque dans le cœur de son appartenance à l'espace nordique, la France voit peser sur elle un doute insupportable quant à la pureté de sa race. Projetant sur la figure du Juif toute l'impureté prétendue de la race française de la même manière qu'il lui attribue les maux de la civilisation occidentale, Drieu perçoit le Français comme un sang-mêlé judaïsé. Comme il ne cesse de le répéter à partir de 1936, si la France se meurt de maladies multiples et incurables, dépopulation, alcoolisme, homosexualité, intellectualité parasite, élites avachies et veules, c'est qu'elle meurt de sa race, qu'elle meurt d'être envahie par les étrangers et déjà conquise par les Juifs. « Nous sommes en train de devenir tous des Juifs $»^{32}$, s'alarme Constant Trubert. Pour Drieu comme pour Drumont, la France juive, héritière d'une Révolution qui n'a profité qu'au peuple juif, est l'ennemi de la France glorieuse de saint Louis, d'Henri IV et de

29. Pierre Drieu La Rochelle, « L'intermède romain », Histoires déplaisantes [1963], Paris, Gallimard, coll. L'Imaginaire, 1988, p. 205.

30. Léon Poliakov, Le Mythe aryen. Essai sur les sources du racisme et des nationalismes, op. cit., p. 39-40. On retrouve des traces de ce traumatisme en particulier au XVIII ${ }^{\mathrm{e}}$ siècle avec la querelle des deux races, gauloise et germanique, puis au $\mathrm{XIX}^{\mathrm{e}}$ siècle avec la valorisation du mythe gaulois (ibid., p. 46-59).

31. Pierre Drieu La Rochelle, Gilles [1939], Paris, Gallimard, coll. Folio, 1973, p. 489.

32. Pierre Drieu La Rochelle, Les Chiens de paille, op. cit., p. 97. 
Louis XIV. C'est son triomphe qui, introduisant l'impureté dans le corps du peuple français et privant la race française de sa vitalité nordique, explique l'évolution de Drieu vers la collaboration. Contre Maurras qui fait l'éloge de l'ordre et de l'harmonie latine héritée de l'Antiquité face à la barbarie germanique et qui manifeste « un goût cruellement étroit de la culture française tout au détriment de la France franque » $^{33}$, son " dégoût des Français », qu'il caractérise lui-même comme « un dégoût de raciste nordique $»^{34}$, pousse Drieu à désirer, pour son salut, le rattachement d'une France abâtardie par le sang méditerranéen et sémite à un Reich allemand promoteur de l'internationale nordique.

C'est de la même manière parce qu'il tient à rattacher la race française à ses origines aryennes qu'il en vient, sans doute à la suite de Schopenhauer, à rapprocher christianisme et paganisme. Le diariste du «Journal d'un délicat » ne cesse de faire des variations autour de l'idée que « les Aryens d'aujourd'hui, qui ont dû devenir antisémites, n'ont pas à rejeter le christianisme parce que sémite. Ils doivent y retrouver leur bien... $»^{35}$. Comme le Walter franquiste de Gilles, Drieu se prononce dans la même logique pour «le catholicisme mâle, celui du Moyen Âge ${ }^{36}$. Ce christianisme raciste, hérité de Gobineau et opposé à la critique nietzschéenne d'un christianisme religion d'esclaves, outre qu'il permet d'écarter l'héritage sémite et par conséquent efféminé du christianisme, devient le fondement d'un nouvel ordre nordique plongeant ses racines dans l'Europe médiévale et ravivant le temps glorieux du saint Empire germanique - nouvel ordre nordique qui, comme l'a bien noté Jacques Lecarme, relève d'un « fascisme » plus « utopique $»^{37}$ que réel.

C'est que le basculement de Drieu vers le fascisme témoigne d'un doute identitaire profond indistinctement social et sexuel et n'autorise donc pas un complet accomplissement de soi. L'homme du Nord n'est

33. Pierre Drieu La Rochelle, « Nouvelle mesure de la France » [septembre 1940, inédit], Chronique politique. 1934-1942, op. cit., p. 242-242.

34. Pierre Drieu La Rochelle, 8 juin 1944, Journal. 1939-1945, Édition établie, présentée et annotée par Julien Hervier, Paris, Gallimard, 1992, p. 385.

35. Pierre Drieu La Rochelle, « Journal d'un délicat » [1944], Histoires déplaisantes, op. cit., p. 34.

36. Pierre Drieu La Rochelle, Gilles, op. cit., p. 658.

37. Jacques Lecarme, Drieu La Rochelle ou le bal des maudits, Paris, PUF, coll. Perspectives critiques, 2001, p. 248. La sensibilité fasciste particulière de Drieu ne contredit toutefois en rien la fermeté de son engagement politique. L'ouvrage de Jacques Lecarme fait sur ce point précis preuve de trop de mansuétude pour l'écrivain. Son objectif légitime, qui est d'affirmer l'intérêt de l'étude de l'œuvre de Drieu malgré ses errements politiques, ne va en effet pas sans une tentation de prendre la défense de l'auteur, en relativisant son engagement fasciste par une attirance plus profonde pour le communisme et en minorant un antisémitisme pourtant réitéré. Pour une discussion plus développée de l'ouvrage de Jacques Lecarme, voir Guillaume Bridet, « Drieu La Rochelle du dadaïsme au fascisme », Mélusine, $\mathrm{n}^{\circ}$ XXIII, « Dedans-Dehors », Études réunies par Henri Béhar, Lausanne, L'Âge d'Homme, 2003, p. 29-30. 
d'abord jamais certain de résister à la tentation néfaste et démobilisante du Sud. Que ce soit avant ou après 1934, le Sud décadent reste pour Drieu un espace dangereux dans la mesure où il tend à retirer toute volonté au ressortissant, plus spécifiquement masculin, du Nord de l'Europe, et à le plonger dans une paresse toute féminine. Quand le soleil brille sur Rome, « tout ce qui [est] vieux » paraît « jeune » à Gille, mais c'est uniquement « pour tromper cet homme venu du Nord $»^{38}$. Alors qu'il séjourne en Andalousie, le diariste du « Journal d'un homme trompé » jouit de « cette simplicité de vie » qui caractérise « les pays chauds », mais il oppose, non sans remords, sa «nonchalance du sud $[\ldots]$ au nord et à ses travaux » et explique qu'il va devoir sous peu retrouver « le Nord, pays des tourments $»^{39}$. Demeurer au Sud, c'est céder à un principe de plaisir dégradant qui empêche l'homme d'œuvrer et de s'assurer un statut social. Le problème est moins le Sud lui-même que la tentation que le Sud exerce sur l'homme du Nord, tentation de la faiblesse le plus souvent liée à son attrait pour une femme dont la sensualité finit par lui ôter toute énergie. «Fille d'un Français d'Algérie et d'une Espagnole », Rose Renard inspire à Camille Le Pesnel un sentiment « enraciné dans la sensualité » qui fait de lui un homme «à jamais malade » et dont la destinée est « irrémédiablement boiteuse $»^{40}$. Non seulement les femmes originaires du Sud sont dangereuses, mais le Sud lui-même pousse à la féminisation de l'homme. Quand Louis Corbin, duc de Friedland, se rend à Bali à bord de son yacht, « les tropiques » lui apportent « une détente si souveraine » qu'il se laisse surprendre par sa femme « aux pieds [...] d'un jeune Russe de bonne extraction $\gg^{41}$ nommé Fédor. L'homme est si bien féminisé par le plaisir des îles australes qu'il découvre alors des penchants homosexuels jusqu'ici insoupçonnés.

L'ascendance nordique intervient comme un moyen imparfait de garantir l'identité de héros romanesques qui, sous ce rapport, sont fort proches de Drieu lui-même se vantant dans sa brève autobiographie d'État civil d'appartenir à 《la race nordique, maîtresse du monde $»^{42}$. Gilles est issu d'un haut lignage. La mer qui baigne les côtes normandes est « ce sein, le premier qui [1']a enivré »; Carentan, son tuteur, dont le nom est identique à celui d'un bourg du Cotentin et qui vit dans une chau-

38. Pierre Drieu La Rochelle, « La voix », Journal d'un homme trompé [1934], Paris, Gallimard, coll. Folio, 1986, p. 71.

39. Pierre Drieu La Rochelle, « Journal d'un homme trompé » [1933], ibid., p. 21.

40. Pierre Drieu La Rochelle, Rêveuse bourgeoisie [1937], Paris, Gallimard, coll. L'Imaginaire, 1995, respectivement p. 102 et p. 104.

41. Pierre Drieu La Rochelle, «La duchesse de Friedland » [1938], Histoires déplaisantes, op. cit., p. 100-101.

42. Pierre Drieu La Rochelle, État civil [1921], Paris, Gallimard, coll. L'Imaginaire, 1977, p. 129. 
mière « entourée de dieux », est « le mystérieux et familier génie de toute sa vie $»^{43}$. Fils de la mer et d'un dieu, de la nature dans sa plus grande force et de l'esprit dans son plein accomplissement, on pourrait s'attendre à ce que Gilles soit, comme Carentan, « un vrai Normand ${ }^{44}$. Le doute ne cesse cependant de le traverser quant à savoir s'il est réellement en droit de revendiquer « cette haute race, sa race. En était-il sûr ? Que savait-il de sa race ? $\gg^{45}$. Son père spirituel peut bien l'initier à la pensée réactionnaire et raciste, il ne parvient pas complètement à le rassurer quant à son identité. Lorsque Dora se promène avec Gilles « dans l'étroite, mais si haute et si noble forêt de Lyons », elle semble l'aimer parce qu'elle respire auprès de lui « le climat nordique »; lorsqu'elle le retrouve au contraire dans le cadre de la vie parisienne, elle le quitte de manière humiliante, selon lui parce qu'il est « latin $»^{46}$. C'est essentiellement face à la force de cette femme nordique que Gilles fait l'expérience de sa propre faiblesse latine, face à sa toute-puissante féminité qu'il devient lui-même un homme châtré, face à son mari confortablement installé dans la vie qu'il prend conscience de son statut social dégradé. Le fils de dieu n'est alors

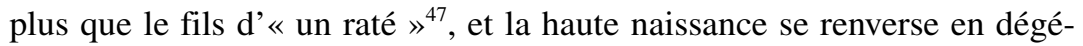
nérescence originelle.

Comme l'apologie du guerrier ou comme celle du chef, l'apologie de la race et, plus spécialement de l'homme nordique, est, pour Drieu, une manière de se donner un père idéal susceptible de garantir l'exaltation de sa propre identité sociale et sexuelle. L'opposition des deux races, juive et nordique, recouvre ainsi toute une série d'oppositions paradigmatiques censées établir un net partage : d'un côté, la laideur, l'aspect nocturne, la passivité, le caractère efféminé, une réussite sociale reposant sur l'imposture ; de l'autre, la beauté, l'aspect diurne et solaire, l'activité, la virilité, une réussite sociale magique reposant sur le génie.

Cette opposition n'est cependant pas si assurée qu'elle ne débouche, à rebours, sur une remise en cause radicale de l'identité. «Ce temps est celui des substitutions : chaque chose est remplacée par son faux $\gg^{48}$. Ici, un personnage passe pour « une espèce de Juif errant», alors qu'il est en fait « Bourguignon $»^{49}$; là, une jeune fille juive prénommée Marianne a

43. Pierre Drieu La Rochelle, Gilles, op. cit., p. 143-145.

44. Ibid., p. 89.

45. Ibid., p. 145.

46. Ibid., respectivement p. 296 et p. 400.

47. Ibid., p. 162 (pour plus de détail sur le personnage de Carentan, voir Jean-François Domenget, « Fascisme et paternité dans Gilles : le personnage de Carentan », dans Marc Dambre et Paul Renard (dir.), Roman 20-50, n 24, «Pierre Drieu La Rochelle », décembre 1997, p. 67-79).

48. Pierre Drieu La Rochelle, L'Homme couvert de femmes, op. cit., p. 175.

49. Pierre Drieu La Rochelle, « Le déserteur », op. cit., p. 223. 
« une sorte de beauté italienne $\aleph^{50}$. On ne peut se fier à personne, et surtout pas à soi-même. Ce règne de l'imposture nationale et raciale généralisée renvoie avant tout à l'imposture première de celui qui aimerait être dieu mais n'est pas sûr d'être quelqu'un. « Inconsciemment, Drieu projette sa propre "décadence" dans sa vision de la nation à laquelle il appartient. Il s'identifie à la France et, en parlant d'elle, il parle encore et toujours de lui $»^{51}$. Comme la France, déchirée entre son appartenance nordique et une race juive qui l'entraîne vers le Sud, Drieu est déchiré entre les identités contradictoires de l'homme et de la femme, du bourgeois en ascension sociale et du bourgeois déclassé. On comprend ainsi pourquoi son imaginaire misogyne, révélé dès L'Homme couvert de femmes en 1925, préfigure précisément son imaginaire antisémite. L'exclusion symétrique des juifs et des femmes, envers négatifs de la virilité et de la dignité, est nécessaire à la préservation d'une identité menacée et pour cela exigeant l'idéalisation compensatoire d'origines nordiques garantes de puissance virile ${ }^{52}$ et de dignité sociale ${ }^{53}$.

50. Pierre Drieu La Rochelle, «L'intermède romain », op. cit., p. 184.

51. Solange Leibovici, Le Sang et l'encre : Pierre Drieu La Rochelle. Une psychobiographie, Amsterdam, Éditions Rodopi, coll. Faux titre, 1994, p. 223.

52. Du côté paternel et, en partie, du côté maternel, Drieu appartient bien à une famille normande (voir Jean Mabire, « Réflexions sur un Coutançais méconnu : Pierre Drieu La Rochelle. I. Drieu, la Normandie et le nordisme », Revue du département de la Manche, t. 3, $\mathrm{n}^{\circ} 11$, juillet 1961, p. 216-220). Il faut croire néanmoins que cette origine ne suffit pas à le rassurer et qu'il a besoin de ce roman familial, repris tout au long de sa vie, pour apaiser un trouble de l'identité sexuelle trouvant sans doute son origine, comme chez d'autres écrivains et penseurs d'extrême droite de la même époque, dans une dévalorisation profonde de la figure paternelle (sur la carence de la figure paternelle entraînant une quête de l'homme fort ; sur l'identification à la figure maternelle à la fois honteuse, puisqu'entraînant une féminisation, et coupable, puisque liée à la réalisation de l'inceste ; sur la projection de l'agressivité adressée aux parents sur les races étrangères comme fondement du racisme et de l'antisémitisme, voir pour Drieu, Solange Leibovici, Le Sang et l'encre : Pierre Drieu La Rochelle. Une psychobiographie, op. cit., p. 90104 ; pour Maurras et Brasillach, Jean-Louis Maisonneuve, L'Extrême droite sur le divan. Psychanalyse d'une famille politique, Paris, Éditions Imago, 1992, p. 66-79 ; pour Rebatet, Lucien Belot, Lucien Rebatet. Un itinéraire fasciste, Paris, Éditions du Seuil, coll. XX ${ }^{\mathrm{e}}$ siècle, 1994, p. 26-27).

53. On peut ici penser, à la suite de Michel Winock traitant plus spécifiquement de l'antisémitisme, que la pensée en terme de peuples et de races constitue, pour Drieu comme pour toute une fraction de la petite bourgeoisie (petits patrons, petits commerçants, rentiers ruinés par l'effondrement des monnaies, etc.), le moyen de réagir à une précarisation grandissante du fait de la crise du système capitaliste, alors que ne se sont encore constituées ni une conscience de classe ni une organisation syndicale à même d'assurer la défense véritable de ses intérêts. La figure glorieuse du nordique, inséparable de la figure inversée et haïe du juif identifié au capitaliste, est une construction mythique, un phénomène de fausse conscience, dont la fonction idéologique est d'apporter à cette petite bourgeoisie un idéal qui ne remette pas en cause l'organisation capitaliste du travail tout en lui permettant d'identifier un coupable. Il faudrait ici revenir sur l'enfance de Drieu et sur les déboires financiers de son père qu'il vécut comme de véritables humiliations personnelles. Par l'intermédiaire de la figure du père, les déterminations mettant à mal la confiance en sa virilité semblent ici très intimement liées à celles mettant à mal son assurance sociale (voir Michel Winock, « Edouard Drumont et la France juive », Nationalisme, antisémitisme et fascisme en France, Paris, Éditions du Seuil, coll. Points, 1990, p. 141-143). 
Ce doute identitaire a des conséquences profondes sur la manière dont, à la lumière de l'idéologie fasciste, Drieu considère sa propre créativité romanesque. Si avant 1934 ses fictions ne sont pas l'exacte illustration du fédéralisme européen et de l'admiration pour le modèle anglais qu'il défend dans ses essais, après cette date elles ne sont pas davantage celle de l'idéologie fasciste.

Drieu ne parvient en effet pas à élever sa prose romanesque jusqu'à l'idéal de poésie mythique qui, non seulement illustre au mieux, mais encore fonde cette idéologie. «Le mythe nazi » de la race nordique est en effet, comme l'expliquent Philippe Lacoue-Labarthe et Jean-Luc Nancy, « le mythe du Mythe, c'est-à-dire le mythe de la puissance créatrice du mythe en général », dans la mesure où il constitue la race nordique ellemême comme «le principe et le lieu d'une puissance mythique $»^{54}$. Cette conception du mythe comme ressource énergétique collective et non plus comme modèle proposé à l'imitation individuelle trouve son origine, non seulement dans les Réflexions sur la violence (1908) de Georges Sorel, mais aussi dans tout le mouvement de renouveau païen inspiré outre-Rhin du Nietzsche falsifié de La Volonté de puissance (1901) et poussant à mettre en avant, contre une Grèce de la mesure qui est celle du classicisme français, une Grèce archaïque et sauvage dont les Allemands seraient les héritiers directs. Participer de la puissance du mythe nordique, c'est détenir la puissance d'une race qui se définit précisément par sa capacité à adhérer activement à son propre mythe et à s'identifier mystiquement à sa mise en œuvre. La caractéristique centrale du national-socialisme est ainsi «la production du politique comme œuvre d'art $»^{55}$, en l'occurrence la production du peuple allemand et de son chef comme une vivante incarnation de l'idéal mythique. L'esthétisation de la politique signifie la priorité accordée à la dimension esthétique sur tout autre critère de jugement, mais surtout la constitution de l'esthétique comme fin ultime de l'action politique et la valorisation de l'artiste comme modèle de l'homme politique.

Paul Sérant a illustré le premier aspect de cette esthétisation en montrant comment le fascisme littéraire consistait en " une poétisation de l'ordre politique et social $»^{56}$. «Le romantisme fasciste » est un romantisme de la race conviant à l'exaltation lyrique des corps et au rassemblement des énergies, au ressourcement originel et à la création d'un nouveau style de vie collective. Il opère comme un dispositif d'embellissement de la réalité sociale par le discours du mythe. Châteaubriant constate avec éblouissement que "l'activité entière de l'Allemagne se nourrit de

54. Philippe Lacoue-Labarthe, Jean-Luc Nancy, Le Mythe nazi, op. cit., p. 57.

55. Ibid., p. 49.

56. Paul Sérant, Le Romantisme fasciste. Étude sur l'œuvre politique de quelques écrivains français, Paris, Fasquelle Éditeurs, 1960, p. 10. 
légendes $\aleph^{57}$; Rebatet se félicite de "l'atmosphère épique » du film de Leni Riefenstahl sur le congrès de Nuremberg en 1934, Le Triomphe de la volonté, atmosphère dans laquelle il perçoit « l'esprit de tout un peuple et de sa politique $»^{58}$.

Comme eux, Drieu se réjouit que les nazis aillent « vers une conception spirituelle, esthétique de la société $»^{59}$. L'homme n'a pas essentiellement besoin de pain, mais d' ' une grande danse méditée $»^{60}$ ou encore « de splendides et enivrantes solennités physiques ${ }^{61}$ lui permettant d'accéder au sens profond de la vie comme à la véritable beauté. Dans la plupart des œuvres romanesques de Drieu, les individus et les peuples ne sont ainsi jamais jugés d'un point de vue moral ou politique sans que vienne se mêler une notation esthétique. Les phalangistes que Walter rencontre en Espagne sont « de beaux gars, jeunes, décidés »; un jeune lieutenant sous les bombes a « un visage transfiguré », c'est-à-dire « un visage d'homme, durement figé ${ }^{62}$. La guerre appartient moins à l'histoire qu'au domaine de l'art, en ce qu'elle est l'occasion par excellence de faire advenir un homme nouveau retrouvant l'idéal de l'athlète grec et du chevalier européen. Le corps réhabilité autorise le plein épanouissement de l'âme; la virilité retrouvée permet d'affronter le danger avec un héroïsme stoïque ; le chant profond de l'amitié virile conduit au renouveau de la race nordique. C'est ainsi que Walter au combat s'identifie glorieusement au « Christ des cathédrales, le grand dieu blanc et viril ${ }^{63}$, et connaît, avant sa mort, la gloire d'une Ascension miraculeuse à la fois spirituelle et esthétique.

Plus encore que ce premier point, qui consiste en une thématisation de l'idéal esthétique nordique, le second point, celui de l'idéalisation de la figure de l'artiste capable de transfigurer la réalité, est tout à fait fondamental, puisqu'il permet de comprendre le regard critique que Drieu porte sur sa propre création romanesque. Être un grand artiste, c'est avant tout, à ses yeux, être un homme fort. La peinture que Dirk Raspe fait d'une prostituée est d'autant plus réussie qu'elle peut donner lieu à un commentaire confondant pratique artistique et assaut guerrier ou amoureux. Dirk, dont le personnage est inspiré à Drieu par Van Gogh, estime que son « imagination [s'est] emparée de cette figure d'un seul coup » et il se

57. Alphonse de Châteaubriant, La Gerbe des Forces (nouvelle Allemagne), Paris, Bernard Grasset, 1937, p. 123.

58. François Vinneuil [Lucien Rebatet], «Les films de Leni Riefenstahl », Je suis partout, 20 août 1937, Cité par Lucien Belot, Lucien Rebatet. Un itinéraire fasciste, op. cit., p. 198-199.

59. Pierre Drieu La Rochelle, « Mesure de l'Allemagne » [NRF, n 246, mars 1934], Socialisme fasciste, Paris, Gallimard, 1934, p. 211.

60. Pierre Drieu La Rochelle, «Écrit dans la rue » [juillet 1934], ibid., p. 104.

61. Pierre Drieu La Rochelle, «L'Esprit du P.P.F. I. Le Parti de la Santé » [L'Émancipation nationale, $\mathrm{n}^{\circ}$ 60, 13 août 1937], Chronique politique. 1934-1942, op. cit., p. 51.

62. Pierre Drieu La Rochelle, Gilles, op. cit., respectivement p. 657 et p. 683.

63. Ibid., p. 687. 
félicite, comme après l'enlèvement d'une tranchée ou d'un jupon, d'avoir montré « de la puissance, de la sûreté » ${ }^{64}$. Aux yeux de Drieu, l'artiste doit être capable de conquérir la réalité et de se l'approprier dans toute sa profondeur pour la révéler à elle-même. L'art opère un travail de transfiguration. «Un vrai peintre » doit être «possédé par le délire de la transposition picturale » et, «dans quelque personnage d'anecdote », il dégage 《un archétype $»^{65}$. On ne s'étonnera pas que Dirk revendique son identité nordique. "Nous autres, gens du Nord, nous portons dans le cœur un soleil inextinguible, un soleil furieux, un soleil qui se dévore lui-même dans un feu incessant et toujours renouvelé, un soleil charrié dans nos veines qui est si terrible qu'il les fait parfois éclater $»^{66}$. L'artiste du Nord, tel un véritable Aryen, a l'âme si large qu'elle s'identifie à celle du soleil, il est le dieu tout-puissant qui nourrit de sa force lumineuse la création, qui du chaos nocturne fait surgir les formes idéales du mythe.

Quand Drieu écrit dans un article que Van Gogh est «l'un des précurseurs de Hitler $»^{67}$, on peut même se demander si le dictateur nazi n'est pas, à ses yeux, le poète par excellence. «Mon œuvre dans sa partie mâle et positive est son incitation et son illustration $»^{68}$, confie-t-il à son journal, comme si cette proximité d'inspiration avec le maître de l'Allemagne nouvelle garantissait la puissance de sa propre créativité. Poète, celui qui se sert de ceux qu'il fascine, membres des défilés de Nuremberg ou soldats partis sur le front des Ardennes, pour exalter l'idéal nordique et parfaire le chef-d'œuvre de sa race, qui insuffle son rêve aux masses rassemblées et, exterminant la souillure du peuple juif, transfigure la matière humaine de son peuple pour l'élever jusqu'à la splendeur archétypale du mythe. À l'opposé du bourgeois rêveur qui manque de force pour affronter l'action et qui se réfugie dans un monde d'illusions chimériques, Hitler est le rêveur d'une rêverie si efficace qu'elle devient la réalité elle-même.

Alors qu'un auteur fasciste comme Brasillach ne doute ni de l'excellence poétique du maître de l'Allemagne, ni de sa propre capacité à participer comme écrivain à son œuvre de transfiguration ${ }^{69}$, Drieu ne

64. Pierre Drieu La Rochelle, Mémoires de Dirk Raspe, Préface de Pierre Andreu, Paris, Gallimard, 1966, respectivement p. 78 et p. 88.

65. Ibid., p. 73.

66. Ibid., p. 221.

67. Pierre Drieu La Rochelle, «Artistes et prophètes » [La Nacion, fin 1939], dans Marc Hanrez (dir.), Pierre Drieu La Rochelle, op. cit., p. 88.

68. Pierre Drieu La Rochelle, 13 mai 1940, Journal. 1939-1945, op. cit., p. 196.

69. Luc Rason montre comment, dans chacun de ses romans, Brasillach substitue au morne réalisme « une collusion du politique et du poétique » (Luc Rason, Littérature et fascisme. Les romans de Robert Brasillach, Paris, Minard, coll. La thésothèque, 1991, p. 91). Dans Les Sept couleurs, roman paru en 1939, le romancier participe lui-même à la transformation du monde réel en monde merveilleux en relayant la parole d'un Hitler dictateur qui, haranguant la foule de Nuremberg, la transfigure magiquement pour la conformer à l'idéal mythique qu'il appelle de ses vœux. 
se croit cependant pas capable de réaliser lui-même un tel programme esthétique.

C'est d'abord son appartenance nationale qui limite sa capacité de transfiguration. La littérature française souffre en effet du mal irrémédiable de ne pas appartenir complètement à cet ensemble nordique seul à même de lui donner toute sa puissance transfiguratrice. Le Français, « incapable de fiction » parce que son rationalisme le confine « dans une vue sèchement sociale de l'homme », s'oppose à l'Anglais, « mystique » tout entier à « sa vision », sachant percevoir «la vie spirituelle qui anime la nature physique » et considérant donc l'homme comme « un animal qui a encore l'âme sauvage de la forêt $»^{70}$. Le premier est tristement réaliste ; le second accède à une transfiguration en laquelle se mêlent le motif de la spiritualité et celui de la sauvagerie, transfiguration oxymorique caractéristique de la conception fasciste de l'homme nouveau, Aryen sorti de la forêt ancestrale pour régénérer, corps et âme, le vieil Occident. La supériorité du poète anglais sur le poète français trouve son origine dans le fait que l'Angleterre est « une île située à la limite des brumes de l'Occident et des frimas du Nord $»^{71}$. Être français et même normand, comme Drieu, c'est au contraire être un poète de moindre envergure, parce qu'on n'appartient pas pleinement à cette race nordique installée en Angleterre et surtout en Allemagne.

Drieu répète ensuite et surtout à de nombreuses reprises que l'art, d'où qu'il soit, connaît une inquiétante décadence : « l'art est en décadence partout sur la planète, en Chine comme en Europe, en Amérique comme en Afrique $»^{72}$. À l'art de son temps, il oppose l'art du Moyen Âge et du $\mathrm{XVII}{ }^{\mathrm{e}}$ siècle français, époques auxquelles il était encore l'expression d'une surabondance et non d'un affaiblissement de la vie, de la force et non du ressentiment. On peut cependant se demander dans quelle mesure ce n'est pas l'art en tant que tel, et non pas seulement l'art des années 1930, qui est marqué, aux yeux de Drieu, d'une essentielle insuffisance. Sommé de parvenir à une transfiguration de la réalité, l'artiste est en effet incapable de parvenir à l'identification effective avec la puissance mythique. « Nous sommes tous, tant que nous sommes, complètement intellectualisés, c'està-dire moribonds $»^{73}$. Si le mythe doit être avant tout la libération instinctive et vécue d'une énergie de la race, la littérature elle-même, du simple fait qu'elle instaure la distance de la pensée, c'est-à-dire à la fois de la raison et de la représentation imaginaire, a nécessairement partie liée avec la

70. Pierre Drieu La Rochelle, «Le poète anglais et le peintre français » [Formes, $\mathrm{n}^{\circ}$ 25, mai 1932], dans Marc Hanrez (dir.), Pierre Drieu La Rochelle, op. cit., p. 61-63.

71. Ibid., p. 60.

72. Pierre Drieu La Rochelle, « Journal d'un délicat », op. cit., p. 53.

73. Pierre Drieu La Rochelle, «Aldous Huxley : À propos d'un roman anglais » $[N R F$, $\mathrm{n}^{\circ}$ 206, novembre 1930], Sur les écrivains, op. cit., p. 98. 
décadence. Il y a un conflit entre l'encre et le sang, la plume et l'épée, entre le littérateur et l'homme d'action, le premier étant toujours accusé de faiblesse et de lâcheté, alors que le second aurait le privilège de la force et du courage. Trop cérébral et manquant d'instinct, l'homme civilisé, et singulièrement celui qui consacre sa vie à la littérature, est un homme atrophié qui ne se replie dans un monde d'idées que parce qu'il est incapable d'agir dans le monde réel. Il est un rêveur sans force qui, contrairement au harangueur nazi, capable de fondre l'individu dans l'irrationalité démiurgique de la foule et du mythe, représente ce dernier sans l'incarner et instaure une dissociation entre l'individu et la vie.

Drieu regrette ainsi de ne pas avoir l'envergure du grand artiste de Berchtesgaden. Privilégiant « la force » et «son rôle indispensable dans la pensée », il reconnaît significativement n'être lui-même qu' « un Hitler avorton $»^{74}$. $S$ 'il cherche à gommer autant qu'il le peut les frontières entre la littérature et la vie au point de nourrir ses œuvres de fiction d'une large part autobiographique, c'est que, pour lui, le roman devrait être un moyen d'agir sur le monde et sur soi. Mais loin de l'efficience fantasmée de la poésie sur la réalité, il perçoit sa propre littérature comme une médiation fâcheuse l'éloignant de son être le plus profond. "Je sais que toute confession de la part d'un contemplatif est une œuvre impie; car elle montre une humanité altérée, fêlée, faussée. Le contemplateur n'est plus un homme, il devait s'abstenir de parler de lui-même $»^{75}$. Si Drieu fait de sa vie la matière de son œuvre, c'est finalement qu'il ne peut faire autrement et qu'il reste attaché à sa personne et à son temps comme une victime de la décadence incapable d'accéder à une transfiguration de la réalité. « Drieu aurait voulu écrire autre chose mais ne le pouvait pas $»^{76}$. Même dans Beloukia et dans L'Homme à cheval, il situe son roman dans un univers exotique, mais il le décrit peu et l'oublie bientôt au profit de ses préoccupations politiques présentes. S'il essaie d'embellir ses personnages en leur apportant « une note de brutalité pittoresque qui les arrache à la médiocrité petite bourgeoise de la fin de la III ${ }^{e}$ République ${ }^{77}$, Bagdad et la Bolivie ne se distinguent pas réellement de la France du temps.

«La littérature n'est qu'une forme édulcorée de la confession, du témoignage qui sont des fonctions éternelles de l'homme, fonctions préalables à l'oraison $\gg^{78}$. Drieu n'atteint que rarement cette seconde étape.

74. Pierre Drieu La Rochelle, « Confession » [hiver 1939-1940], dans Marc Hanrez (dir.), Pierre Drieu La Rochelle, op. cit., respectivement p. 94 et p. 96.

75. Ibid., p. 96.

76. Solange Leibovici, Le Sang et l'encre : Pierre Drieu La Rochelle. Une psychobiographie, op. cit., p. 9.

77. Julien Hervier, Deux individus contre l'histoire : Drieu La Rochelle et Ernst Jünger, op. cit., p. 196.

78. Pierre Drieu La Rochelle, « Journal d'un délicat », op. cit., p. 51. 
Non seulement il laisse peu de place à la transfiguration et ne parvient pas à faire de la littérature autre chose qu'un miroir de la réalité, mais encore il fait d'elle un miroir déformant et qui tend à la caricature. L'oraison censée élever l'homme vers le divin ou vers l'exaltation du mythe nordique reste prisonnière d'une confession constituant pour la plus grande part, comme il le reconnaît lui-même, « une œuvre de satire » mettant en procès « la décadence $»^{79}$ et, comme en témoignent les incarnations successives de sa propre personne dans le personnage de Gille(s), faisant également une large place à l'auto-dénigrement. Drieu ne sera pas le poète jaculatoire, créateur continu et tout-puissant de la réalité sociale et de sa propre réalité ; il ne sera pas le dieu forestier en état de transe communiant avec le divin et se retrempant dans la pureté originelle. Il sera, non sans ressentiment et comme le prouve encore la parution récente chez Gallimard des Notes pour un roman sur la sexualité, le chroniqueur désenchanté d'une décadence universelle qui ne l'épargne pas lui-même.

Drieu n'est pas suffisamment convaincu par l'idéologie raciste pour se contenter de s'en faire le simple propagandiste. C'est sans aucun doute l'une des raisons pour lesquelles il n'écrit pas uniquement des essais ou des articles de presse politiques et éprouve régulièrement le besoin de recourir à des fictions romanesques qui, très largement autobiographiques, sont autant de moyens pour tenter de mettre un terme au doute identitaire qui le frappe en reconstruisant une identité idéale. L'imaginaire racial est ainsi profondément lié à une recherche esthétique et à une démarche créatrice qui, dans son cas, constituent cependant une tentative ratée de réparation narcissique. Drieu ne passe à peu près jamais, et surtout pas en ce qui le concerne, du rêve fasciste de l'esthétisation de soi par l'exaltation de la pureté raciale à la croyance d'une esthétisation effective. Si le normand dégénéré n'apparaît pas à ses propres yeux comme un nordique conquérant, c'est qu'il y a une contradiction insurmontable entre le fait d'être romancier et celui d'être un guerrier nordique. Le mot à mot n'est pas un corps à corps. Drieu ne se considère pas comme un écrivain apportant une aide efficace à l'homme d'action nazi ; il ne se considère pas non plus et encore moins comme un homme d'action. C'est peut-être ce doute permanent et ce sentiment d'échec qui, brisant l'enfermement dans la folle logique du mythe raciste, le transforma en bourreau de lui-même : Drieu la plaie, Drieu le couteau, Drieu au matin du 16 mars 1945 — « long type triste »asphyxié par le gaz et, Sartre l'avait bien vu, par « la haine de soi ${ }^{80}$.

79. Pierre Drieu La Rochelle, Préface de Gilles, op. cit., p. 10.

80. n.s. [Jean-Paul Sartre], «Drieu la Rochelle ou la haine de soi », Les Lettres françaises, $\mathrm{n}^{\circ} 6$, avril 1943 , p. 3 . 